\title{
Solar cycle signatures in the NCEP equatorial annual oscillation
}

\author{
H. G. Mayr ${ }^{1}$, J. G. Mengel ${ }^{2, \dagger}$, F. T. Huang ${ }^{3}$, and E. R. Nash ${ }^{2}$ \\ ${ }^{1}$ NASA Goddard Space Flight Center, Greenbelt, MD, USA \\ ${ }^{2}$ Science Systems and Applications Inc., Lanham, MD, USA \\ ${ }^{3}$ University of Maryland, Baltimore, MD, USA \\ ${ }^{\dagger}$ deceased
}

Received: 20 May 2009 - Revised: 15 July 2009 - Accepted: 5 August 2009 - Published: 19 August 2009

\begin{abstract}
Our analysis of temperature and zonal wind data (1958 to 2006) from the National Center for Atmospheric Research (NCAR) reanalysis (Re-1), supplied by the National Centers for Environmental Prediction (NCEP), shows that the hemispherically symmetric 12 -month equatorial annual oscillation (EAO) contains spectral signatures with periods around 11 years. Moving windows of 44 years show that, below $20 \mathrm{~km}$, the 11-year modulation of the EAO is phase locked to the solar cycle (SC). The spectral features from the 48-year data record reveal modulation signatures of 9.6 and 12 years, which produce $\mathrm{EAO}$ variations that mimic in limited altitude regimes the varying maxima and minima of the $10.7 \mathrm{~cm}$ flux solar index. Above $20 \mathrm{~km}$, the spectra also contain modulation signatures with periods around 11 years, but the filtered variations are too irregular to suggest that systematic SC forcing is the principal agent.
\end{abstract}

Keywords. Meteorology and atmospheric dynamics (Climatology; Middle atmosphere dynamics; Waves and tides)

\section{Introduction}

\subsection{Solar cycle effects}

In several papers, solar cycle (SC) effects have been linked to the quasi-biennial oscillation (QBO), which dominates the zonal winds of the lower stratosphere at equatorial latitudes. One SC connection was brought out by Labitzke $(1982,1987)$ and Labitzke and van Loon $(1988,1992)$, who discovered that the temperatures at northern polar latitudes in winter are positively and negatively correlated with the $\mathrm{SC}$ when the QBO is in its negative and positive phase respectively; and this correlation was confirmed by Dunker-

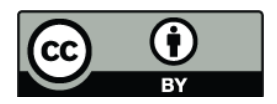

Correspondence to: H. G. Mayr (hans.g.mayr@nasa.gov) ton and Baldwin (1992), and Baldwin and Dunkerton (1998). Inserting rocketsonde data to simulate the QBO, Matthes et al. (2004) reproduced quantitatively the observed SC variations in the polar stratosphere. The observed SC effect was also reproduced reasonably well by Palmer and Gray (2005), who employed the gravity wave parameterization of Hines $(1997 \mathrm{a}, \mathrm{b})$ to simulate the downward phase progression of the QBO.

Related to the present study, Salby and Callaghan (2000) showed that the SC modulates the QBO itself. In the power spectrum from their 41-year data record, a sharp peak at 0.41 cycles per year (cpy) describes the mean QBO of about 29 months, and smaller neighboring maxima at 0.5 and 0.59 cpy reveal frequencies representing the 11-year SC and its second harmonic of 5.5 years. Salby and Callaghan synthesized these spectral features to produce the filtered SC modulation of the QBO embedded in the data, which shows at $20 \mathrm{~km}$ zonal wind variations from about 12 to $20 \mathrm{~m} / \mathrm{s}$ that are correlated with the $10.7 \mathrm{~cm}$ flux. Analyzing 50 years of wind measurements, Hamilton (2002) confirmed the large QBO modulation inferred by Salby and Callaghan (2000) but concluded that the SC connection is not as clear in the extended data record. With data supplied by the National Centers for Environmental Prediction (NCEP), Salby and Callaghan (2006) demonstrated that the stratospheric QBO temperatures at low latitudes vary with the SC.

With a 2-D model extending from about 15 to $30 \mathrm{~km}$, Cordero and Nathan (2005) simulated the observed SC modulation of the QBO, which is driven with prescribed Kelvin waves and Rossby gravity waves. In this model, the SC effects on ozone influence the wave interaction to generate variations of the QBO amplitude that are in phase with the observed zonal winds reported by Salby and Callaghan (2000). Mayr et al. (2006) simulated the observed SC effect with a 3-D model, which relies primarily on parameterized gravity waves $(\mathrm{GW})$ to produce the QBO. In this model, the SC influence is transferred to the QBO by a hemispherically

Published by Copernicus Publications on behalf of the European Geosciences Union. 
symmetric equatorial annual oscillation (EAO) that acts as pacemaker (Mayr et al., 2005).

\subsection{Equatorial oscillations}

We believe that the unique dynamical conditions around the equator play a pivotal role in generating the large SC effects associated with the QBO. As Lindzen and Holton (1968) emphasized, the wave forcing, which generates the $\mathrm{QBO}$, is very effective around the equator where the Coriolis force vanishes. Without the meridional winds, the momentum source is dissipated only by viscosity. Away from the equator, the meridional circulation increasingly comes into play to redistribute and dissipate the flow oscillation, in part through radiative cooling. This property is displayed in our model results, generated with latitude independent wave source, which produces a QBO that still peaks at the equator (Mengel et al., 1995); and Haynes (1998) provided an analytical understanding of the process involved.

In generating the QBO, an important factor is the nonlinear nature of the wave mean flow interactions characterized by critical level absorption. The positive feedback in the momentum source amplifies the oscillation, and this process is very effective because the QBO zonal winds are hemispherically symmetric to first order. Waves propagating up around the equator then generate oscillations that are in phase across the region to reinforce each other, in contrast to the situation for the anti-symmetric component where opposite interactions in the two hemispheres tend to cancel each other. Generated by wave mean flow interactions, the symmetric QBO slowly propagates down through the stratosphere, in contrast to the anti-symmetric component that reveals virtually no phase progression with height.

The above-discussed properties of the QBO appear also in the equatorial annual oscillation (EAO) identified in stratospheric NCEP data (Mayr et al., 2007b). Like the QBO, the EAO peaks at the equator and slowly propagates down through the stratosphere. The EAO is part of a global-scale annual oscillation (AO) that is hemispherically symmetric, which has its primary peak at about $60^{\circ}$ latitude (see Fig. 1c of Mayr et al., 2007b). This symmetric AO is distinctly different from the dominant $\mathrm{AO}$, which is hemispherically antisymmetric (vanishing at the equator) and is generated to first order by the differences in solar heating associated with the Earth's orbital inclination.

The symmetric AO, of which the EAO is the equatorial portion, can be generated by solar heating under the influence of the hemispheric differences associated with topography. Commenting on the above NCEP data analysis, Klemens Hocke (University of Bern) pointed out to us that such an $\mathrm{AO}$ can be generated by the Earth's orbital eccentricity around the Sun. Furthermore, a reviewer pointed out that a significant 12-month AO signature was found in the EUV radiation during the last solar cycle (Hocke, 2008).
Considering that the observed stratospheric SC effects are closely connected to the QBO, we asked the question whether the EAO might also harbor such signatures. Since solar heating generates primarily the 12-month AO, it would be natural to find that the SC modulates the EAO. Furthermore, one may expect that the SC signature of the EAO, like that of the QBO, is amplified by wave interactions at equatorial latitudes, as was shown in a modeling study (Mayr et al., 2007a).

In the following, we present evidence that the EAO in the NCEP/NCAR Re-1 zonal wind and temperature data reveals signatures that could be generated by the SC.

\section{NCEP/NCAR Re-1 zonal wind and temperature analyses}

In our study, we analyze for the years from 1958 to 2006 the data from the National Center for Atmospheric Research (NCAR) reanalysis (Re-1) (Kalney et al., 1996), supplied by the National Centers for Environmental Prediction (NCEP). The zonal-mean temperatures and zonal winds are employed, which are spaced at 2.5 degrees in latitude and extend from the surface to $10 \mathrm{hPa}$ (approximately $31 \mathrm{~km}$ ). As discussed by Kalney et al. (1996) and Kalney (2003), the Re-1 data represent mainly balloon-borne radiosonde observations that have been assimilated in GCM simulations. Nadir sounding satellite measurements introduced after 1978 improved the global coverage, but Huesmann and Hitchman (2001, 2003) showed that the observations produced a jump in the NCEP/NCAR Re-1 temperatures, in particular near the tropopause and at around $30 \mathrm{~km}$.

Following Mayr et al. (2007b), the NCEP/NCAR Re-1 data are delineated in terms of their hemispherically symmetric and anti-symmetric components. For this purpose, the data in the Northern and Southern Hemispheres, $D_{n}$ and $D_{s}$, respectively, are split into the symmetric (S) and anti-symmetric (A) components by computing respectively $\mathrm{DS}_{n s}=\left(D_{n}+D_{s}\right) / 2$ and $\mathrm{DA}_{n}=\left(D_{n}-D_{s}\right) / 2, \mathrm{DA}_{s}=\left(D_{s}-D_{n}\right) / 2$. The $\mathrm{DS}_{n s}$ component then describes the symmetric AO (and QBO), which peak at the equator, while $\mathrm{DA}_{n}=-\mathrm{DA}_{s}$ describes to first order the dominant anti-symmetric AO with opposite phase in the two hemispheres, which vanishes at the equator.

\subsection{Moving window 11-year modulations of symmetric AO}

For the years from 1958 to 2006 , the $10.7 \mathrm{~cm}$ flux varies on average with a period of about 11 years. (As shown in Fig. 7a below, the maxima occur near 1958, 1969, 1980, 1990, 2001.) To reveal the 11-year variations of the symmetric AO, moving data windows of 44 years are analyzed. Applying Fourier analysis to the NCEP/NCAR Re-1 zonal wind data for the initial 44-year time span from 1958 to 2002, 

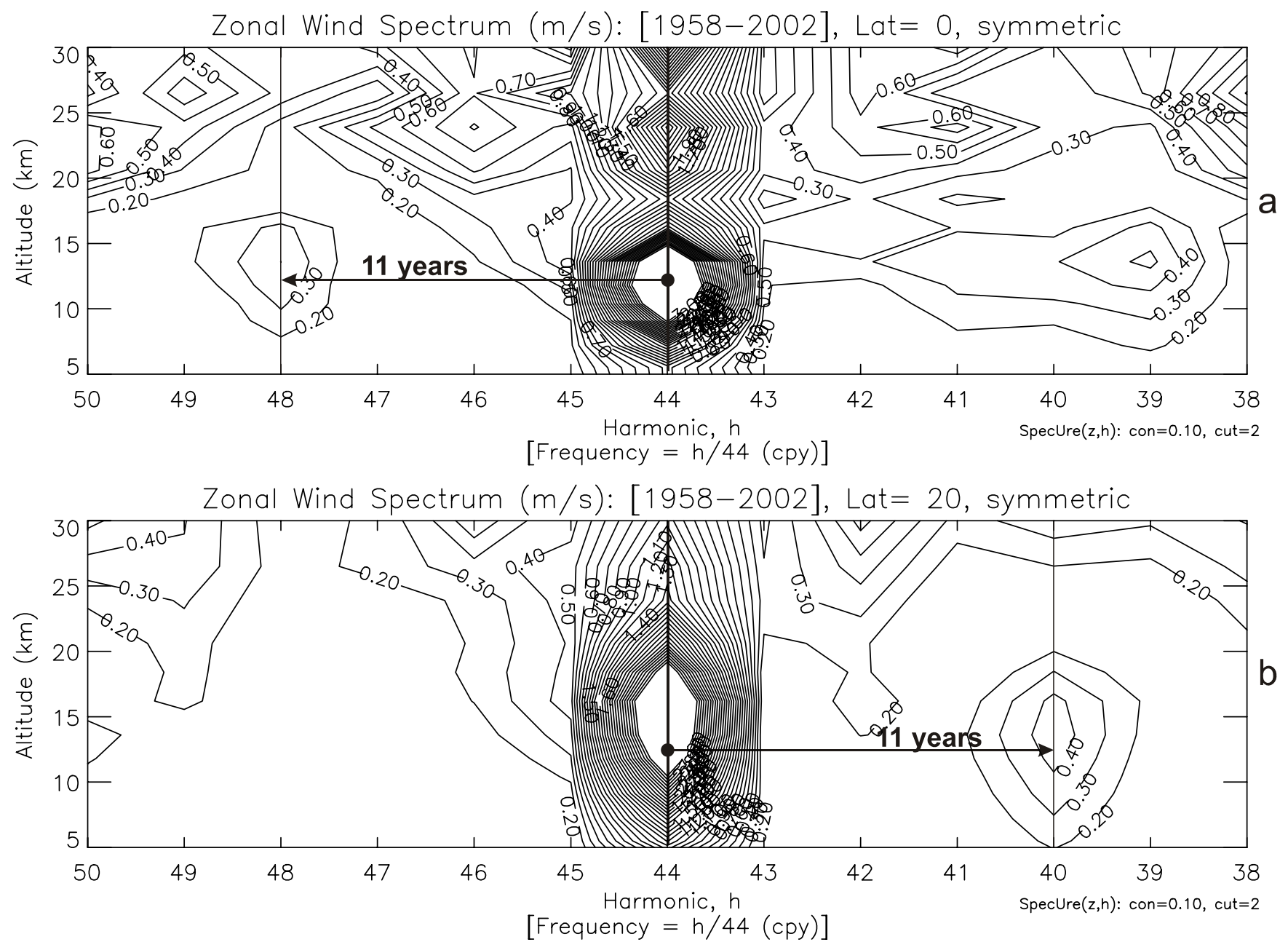

Fig. 1. Spectra covering 44 years from 1958-2002 for the hemispherically symmetric NCEP/NCAR Re-1 zonal winds at the equator (a) and $20^{\circ}$ latitude (b). Amplitudes are plotted versus Fourier harmonics, h, and altitude. The corresponding frequencies are then h/44 (cycles per year, cpy), and the periods are $44 / \mathrm{h}$ (years). With contour intervals of $0.1 \mathrm{~m} / \mathrm{s}$, the 12 -month annual oscillation $(\mathrm{AO})$ at $\mathrm{h}=44 \mathrm{is}$ saturated. Weak signatures of the 11-year modulation $(h=4)$ are evident at $h=48(44+4)$ in (a), and at $h=40(44-4)$ in (b). The spectra for the subsequent 44-year windows, starting in 1959 and shifted by one year, show similar features.

we present in Fig. 1 the symmetric amplitude spectra at the equator (Fig. 1a) and at $20^{\circ}$ latitude (Fig. 1b), which are plotted in terms of the harmonics, $h$. The frequencies are then $\mathrm{h} / 44$ (cycles per year), and the periods are $44 / \mathrm{h}$ (years). With the applied small contour intervals of $0.1 \mathrm{~m} / \mathrm{s}$, the spectral features for the 12-month $\mathrm{AO}$, at $\mathrm{h}=44$, are saturated. In Fig. 1a, the spectral feature at $\mathrm{h}=48(44+4)$ is separated by 4 wave numbers from the $\mathrm{AO}$, and this is the signature of the 11-year modulation as indicated with the arrow. A pronounced 11-year signature is also evident at $\mathrm{h}=(44-4)$ in Fig. 1b. These spectral side lobes describe amplitude modulations produced by nonlinear processes, which essentially multiply the dominant anti-symmetric $\mathrm{AO}, \operatorname{Aexp}\left(\mathrm{it} \omega_{A}\right)$, with the anti-symmetric 11-year SC forcing, $\operatorname{Sexp}\left(i t \omega_{S}\right)$, to produce symmetric oscillations, $\operatorname{ASexp}\left(\operatorname{it}\left(\omega_{A} \pm \omega_{S}\right)\right)$, with frequencies, $\omega_{A} \pm \omega_{S}$, and related harmonics, $\mathrm{h}_{A} \pm \mathrm{h}_{S}$. When the analysis is applied to the subsequent running 44-year windows, starting from 1959 to 1962 and spaced by one year, the spectra reveal 11-year signatures similar to those shown in Fig. 1 - which supports the SC interpretation.

To test the SC hypothesis, we need to synthesize the spectral features involving the $\mathrm{AO}$ and its 11-year side lobes. Considering the relatively weak side lobes in relation to the $\mathrm{AO}$, however, the modulation would be difficult to discern. To get around this problem, we portray the modulation pattern specifically and separate from the magnitude of the AO that is being modulated, as illustrated in Fig. 2, and in Fig. 4 of Mayr et al. (2008). In Fig. 2a, we synthesize the equatorial zonal wind variations at $13.6 \mathrm{~km}$, employing the spectral features from Fig. 1a for $\mathrm{h}=44$ and $44 \pm 4$ (although not pronounced, the amplitudes at $\mathrm{h}=40$ are included since nonlinear interactions could generate them in principle). The 


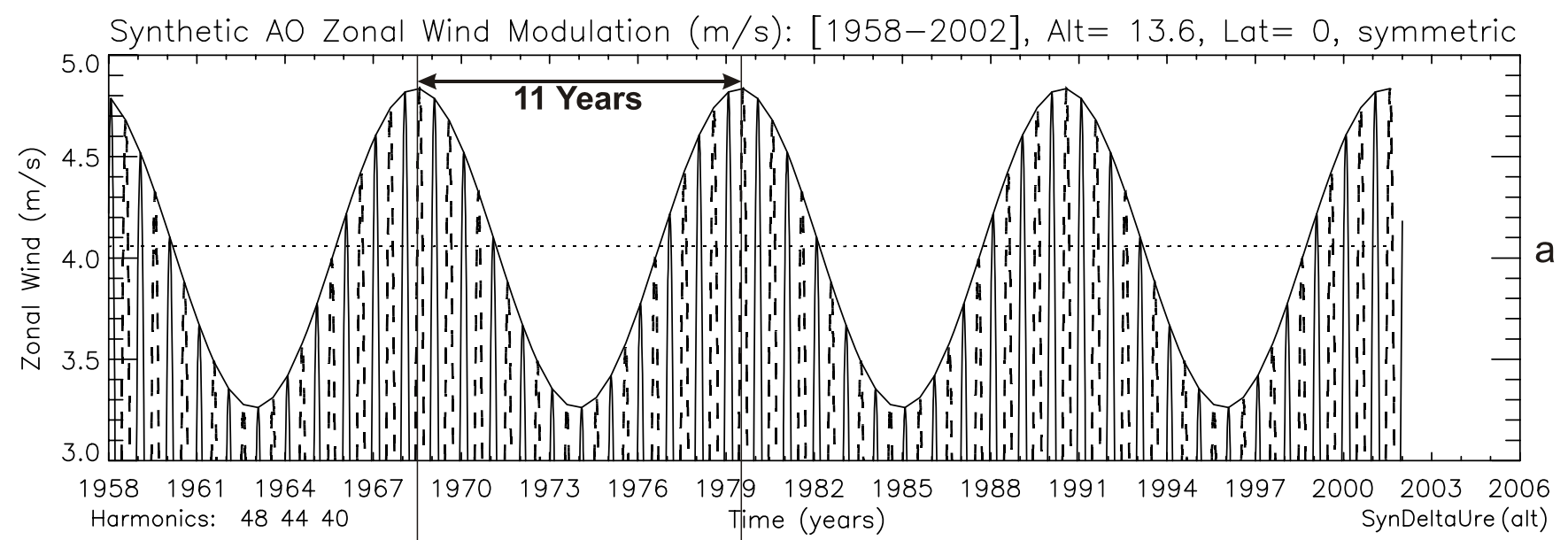

Synthetic AO Zonal Wind Modulation (m/s): [1958-2002], Lat=0, symmetric

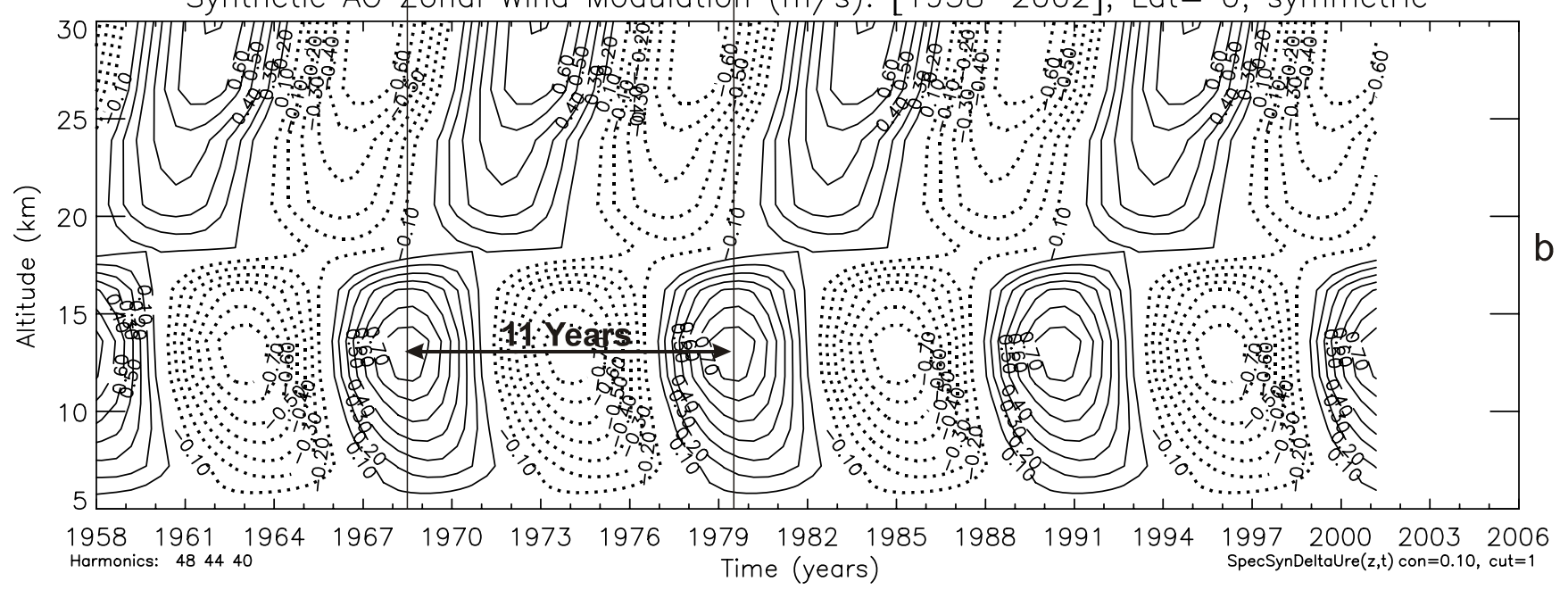

Fig. 2. (a) Synthesis of zonal wind variations near $14 \mathrm{~km}$, employing $\mathrm{h}=44$ with $44 \pm 4$, shows that the 11 -year modulation is small compared with the amplitude of the AO. The positive and negative wind excursions are presented respectively with solid and dashed lines, and the solid line represents the envelope that portrays the modulation. The modulated zonal winds vary by less than $1 \mathrm{~m} / \mathrm{s}$ around a value of about $4 \mathrm{~m} / \mathrm{s}$ (dotted line). (b) This small modulation is presented as contour plot versus altitude and time to describe the 11-year zonal wind variation of the symmetric AO. It shows that at altitudes around $14 \mathrm{~km}$ the maxima and minima have magnitudes of about $0.75 \mathrm{~m} / \mathrm{s}$, commensurate with the modulation excursion close to $1.5 \mathrm{~m} / \mathrm{s}$ in (a). The oscillation pattern recurs in 11-year intervals throughout the entire 44-year time span.

resulting annual oscillations are presented for the positive phase (solid) and negative phase (dashed), to show that the zonal winds vary from about 3.3 to $4.8 \mathrm{~m} / \mathrm{s}$. The envelope of this modulation (solid line) is computed from the maxima of the positive and negative AO zonal wind excursions, and the dotted line at about $4 \mathrm{~m} / \mathrm{s}$ represents the time average of the envelope. The deviations of this envelope from the mean, about $\pm 0.75 \mathrm{~m} / \mathrm{s}$, then describe the magnitude of the modulation. Applying this procedure for all the altitudes from 5 to $30 \mathrm{~km}$, we construct the contour plot (Fig. 2b) that displays the SC modulation of the zonal wind $\mathrm{AO}$ on altitude versus time coordinates. In agreement with Fig. 2a, it shows that the symmetric $\mathrm{AO}$ at about $14 \mathrm{~km}$ is modulated by about $1.5 \mathrm{~m} / \mathrm{s}$, with the maximum (positive) occurring near 1958, and the minimum (negative) near 1963. Unaffected by the magnitude of the AO amplitude, Fig. $2 \mathrm{~b}$ reveals the modulation pattern, which recurs in 11-year intervals throughout the entire analyzed time span of 44 years.

For the $\mathrm{SC}$ to be involved in generating the 11-year signatures in the spectra, the variations are required to be phase locked, and this is shown in Fig. 3. For running windows, with one-year intervals, we present in Fig. 3a at the equator the synthesized zonal wind variations, employing $\mathrm{h}=44$ and $44 \pm 4$. Since the modulation patterns repeat in 11-year intervals (Fig. 2), the displayed time span is restricted to the years between 1985 and 1997. (The first panel in Fig. 3a corresponds to Fig. 2b.) This shows that, at altitudes below about $17 \mathrm{~km}$, the modulation patterns are similar in amplitude and phase. For the moving windows, the localized maxima around $14 \mathrm{~km}$ occur at the equator (Fig. 3a) near the year 

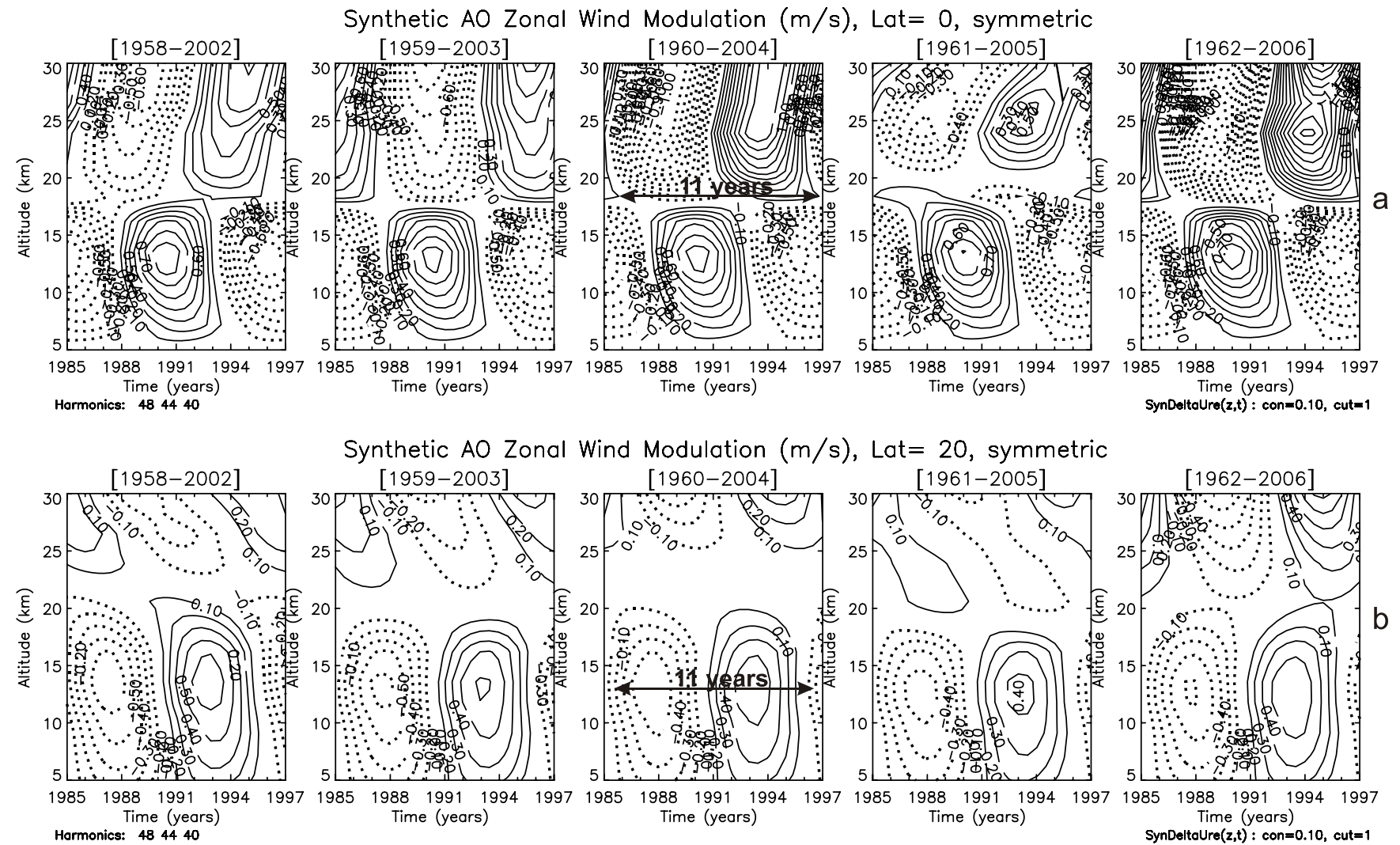

Synthetic AO Zonal Wind Modulation ( $\mathrm{m} / \mathrm{s})$, Lat $=20$, symmetric
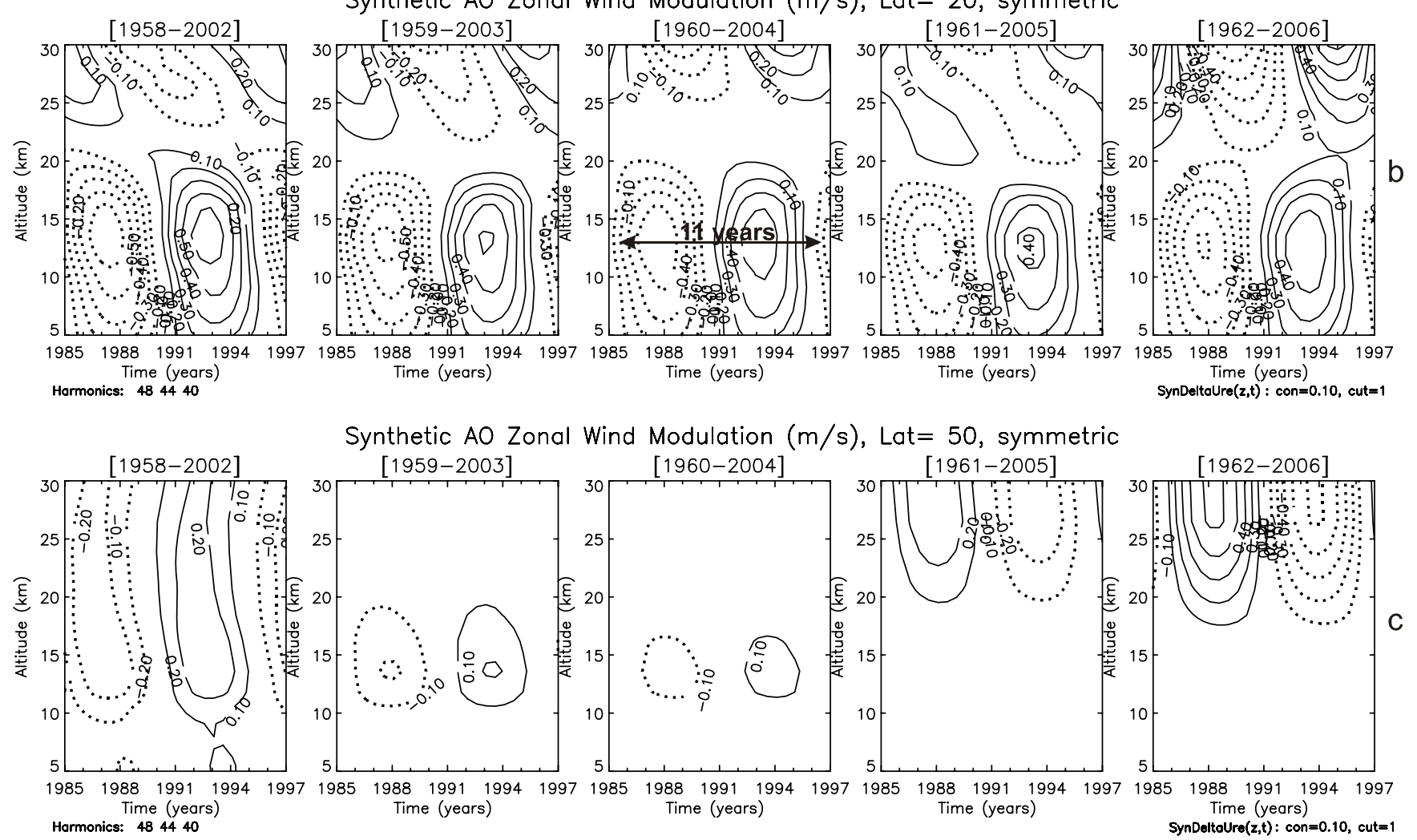

Fig. 3. Synthesized zonal wind modulations of the symmetric AO for the moving 44-year time intervals beginning in 1958 and ending in 2002 at the equator (a), and at $20^{\circ}$ (b) and $50^{\circ}$ (c) latitudes. The harmonics $\mathrm{h}=44$ and $44 \pm 4$ are synthesized, like in Fig. $2 \mathrm{~b}$. Since the oscillation patterns repeat in 11-year intervals, the results are shown only for the years from 1985 to 1997 . At the equator (a), the 11-year modulation peaks between 10 and $15 \mathrm{~km}$, and the maximum occurs near 1990 in each of the windows, which indicates that the variations are phase locked due to potential forcing by the SC. The modulation pattern at higher altitudes, however, varies in amplitude and phase, indicative of a less systematic source. At $20^{\circ}$ latitude (b), and below $20 \mathrm{~km}$, systematic 11-year zonal wind modulations are generated with smaller amplitudes; and at mid latitudes (c), the modulations all but disappear.

1990, close to the peak of the $10.7 \mathrm{~cm}$ flux, and they occur at $20^{\circ}$ latitude (Fig. 3b) in 1993. Above $20 \mathrm{~km}$, however, the patterns vary considerably to indicate a lack of systematic forcing. As shown in Fig. 3, the 11-year modulation of the symmetric $\mathrm{AO}$ peaks at the equator and all but disappears at $50^{\circ}$ latitude (Fig. 3c).
As seen from Fig. 4, 11-year signatures also appear in the symmetric NCEP/NCAR Re-1 temperature variations. For the 44-year time span from 1958 to 2002, symmetric amplitude spectra are shown. With the applied small contour intervals of $0.02 \mathrm{~K}$ at the equator (Fig. $4 \mathrm{a}$ ) and $0.04 \mathrm{~K}$ at the pole (Fig. 4b), the spectral features for the 12-month $\mathrm{AO}$ at $\mathrm{h}=44$ 

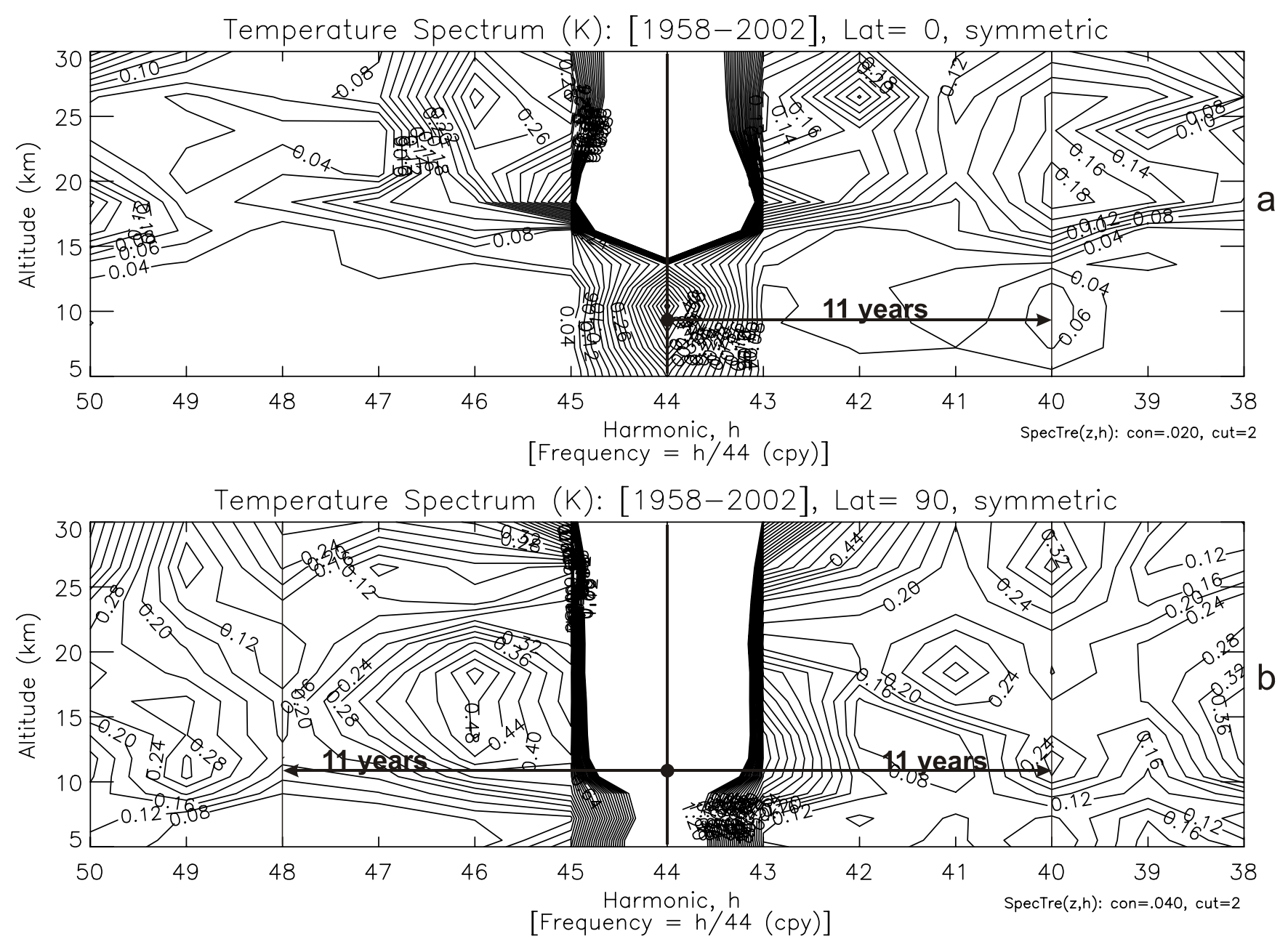

Fig. 4. Amplitude spectra are shown, covering 44 years from 1958-2002 for the hemispherically symmetric NCEP/NCAR Re-1 temperatures at the equator (a) and pole (b). With contour intervals of $0.02 \mathrm{~K}$ (a) and $0.04 \mathrm{~K}$ (b), the 12 -month oscillation amplitudes at $\mathrm{h}=44 \mathrm{are}$ saturated. Weak signatures of the 11-year modulation are evident at $h=40$ (44-4) in (a) and at $h=44 \pm 4$ in (b). The spectra for the subsequent 44 -year windows, starting in 1959 and shifted by one year, show similar features.

are saturated. In Fig. 4a, an isolated signature appears at $10 \mathrm{~km}$, and less pronounced larger amplitudes are generated above $14 \mathrm{~km}$. At the pole (Fig. 4b), relatively large and distinct 11 -year features appear at $10 \mathrm{~km}$ and above $25 \mathrm{~km}$. The spectra for the subsequent 44-year running windows, starting from 1959 to 1962, are similar. And this indicates, like the zonal wind data, that the SC forcing may be involved in generating the spectral side lobes.

Analogous to Fig. 3, we present in Fig. 5 the synthesized (filtered) temperature variations. At the equator (Fig. 5a), the modulation patterns are similar in amplitude and phase near $15 \mathrm{~km}$ and below. The localized maximum between 15 and $20 \mathrm{~km}$ occurs during the year 1992, and the one near $10 \mathrm{~km}$ occurs in 1993. Above $20 \mathrm{~km}$, in contrast, the patterns vary considerably to indicate that systematic forcing does not prevail. At the pole (Fig. 5b), systematic 11-year temperature modulations are evident around $10 \mathrm{~km}$, where such a signa- ture is also seen in the spectrum (Fig. 4b). Except for the first panel (1958-2002), the maxima of the temperature modulations occur in 1994 for the remaining four time spans, again indicating that the modulations are phase locked and could be generated by the SC. As in Fig. 5a at the equator, the oscillation patterns at higher altitudes however vary erratically to indicate that the 11-year signatures are not caused by a systematic SC source.

\subsection{Variable solar cycle signatures related to F10.7}

For the time span 1958 to 2006 of the NCEP/NCAR Re1 data discussed, the dominant Fourier harmonics of the F10.7 solar proxy are $\mathrm{h}=5$ and 4 (periods of 12 and 9.6 years), as shown in Fig. 6a. These periods also appear in the NCEP/NCAR Re-1 spectra for the zonal winds at the equator (Fig. 6b), and for the temperatures at the equator (Fig. 6c) and 

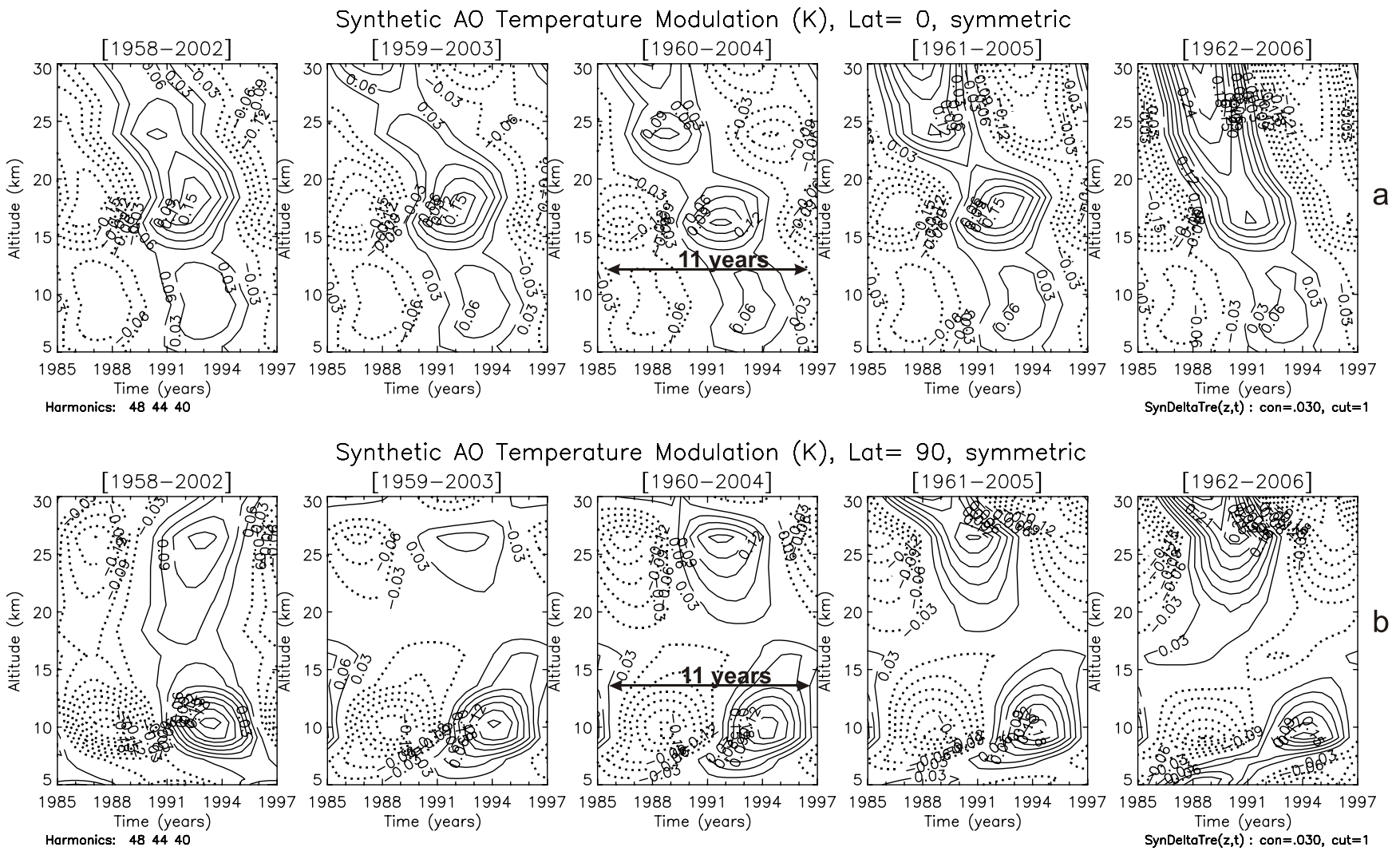

Synthetic AO Temperature Modulation (K), Lat $=90$, symmetric
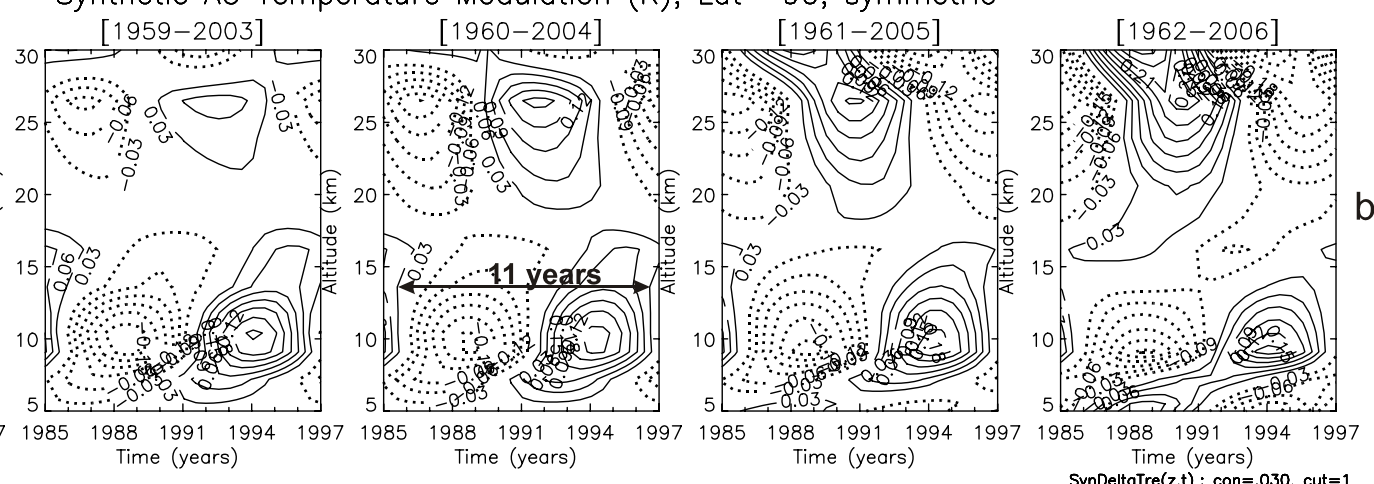

Fig. 5. Analogous to Fig. 3, synthesized temperature modulations of the symmetric AO for the moving 44-year time intervals at the equator (a), and pole (b). At the equator (a), the 11-year modulation peaks between 15 and $20 \mathrm{~km}$, and the maximum occurs near 1992 in each of the windows. This indicates that the modulation is phase locked, possibly due to systematic forcing by the SC. This pattern is also apparent below $15 \mathrm{~km}$, but the variations at higher altitudes are erratic. (b) At the pole, systematic 11-year temperature modulations occur around $10 \mathrm{~km}$ altitude, consistent with the corresponding signature in Fig. $4 \mathrm{~b}$.

pole (Fig. 6d). As in Fig. 1 for the 44-year moving windows, the zonal wind signatures are most pronounced between 10 and $15 \mathrm{~km}$. In the equatorial temperatures (Fig. 6c), these periods appear mainly between 15 and $25 \mathrm{~km}$, where the average 11-year modulation is also seen in Fig. 4a. At the pole (Fig. 6d), the 12-month modulation is mainly excited.

In Fig. 7a we present the monthly $10.7 \mathrm{~cm}$ flux from 1958 to 2006 together with a synthesis of the dominant harmonics $(h=4$ and 5). The filtered variations reveal a distinct pattern of variable maxima and minima. The largest maximum occurs near 1980, which is followed by the one in 1991, and both are straddled by the weaker maxima around 1970 and 2001.

As shown in Figs. 3 and 5, for certain altitude regimes, the 11-year modulations of the $\mathrm{AO}$ zonal winds and temperatures are phase locked in five consecutive data windows. In these regions of the atmosphere, Fig. 7 shows that the filtered modulations partially mimic the variability of the solar cycle in Fig. 7a. Commensurate with the format in Fig. 2b, we present in Fig. 7b for the 48-year NCEP/NCAR Re-1 data a synthesis of the zonal wind harmonics, $\mathrm{h}=48$ with $(48 \pm 4)$ and (48 \pm 5 ), to account for the 12 and 9.6-year modulations of the symmetric $\mathrm{AO}$ at the equator. Consistent with the results in Fig. 3a, a coherent modulation pattern is evident below about $18 \mathrm{~km}$. Near $14 \mathrm{~km}$, where the largest amplitudes are generated in this altitude regime $(\sim \pm 1 \mathrm{~m} / \mathrm{s})$, we also show with heavy solid line the filtered wind modulations, which reproduce remarkably well the solar cycle variability in Fig. 7a. With almost constant phase difference, the variable maxima and minima in the NCEP/NCAR Re-1 zonal winds mimic those of the solar cycle. Above $18 \mathrm{~km}$, and consistent with Fig. 3a, the modulation pattern in Fig. $7 \mathrm{~b}$ is highly variable, and it has no resemblance to the solar flux index (Fig. 7a).

The filtered equatorial $\mathrm{AO}$ temperature modulations in Fig. 7c also reveal the variable pattern of the solar cycle but only at altitudes around $16 \mathrm{~km}$ (heavy line). This altitude is in the regime of the phase-locked 11-year oscillations shown in Fig. 5. The temperature maxima occur almost exactly 2 years later than those for the zonal winds near $14 \mathrm{~km}$ (Fig. 7b). At altitudes above $18 \mathrm{~km}$, the filtered temperature modulations differ significantly from the solar cycle pattern. Around $10 \mathrm{~km}$ and below, a weak temperature modulation is generated, which has a period of about 12 years. 
F10.7 Amplitude Spectrum: [1958-2006]
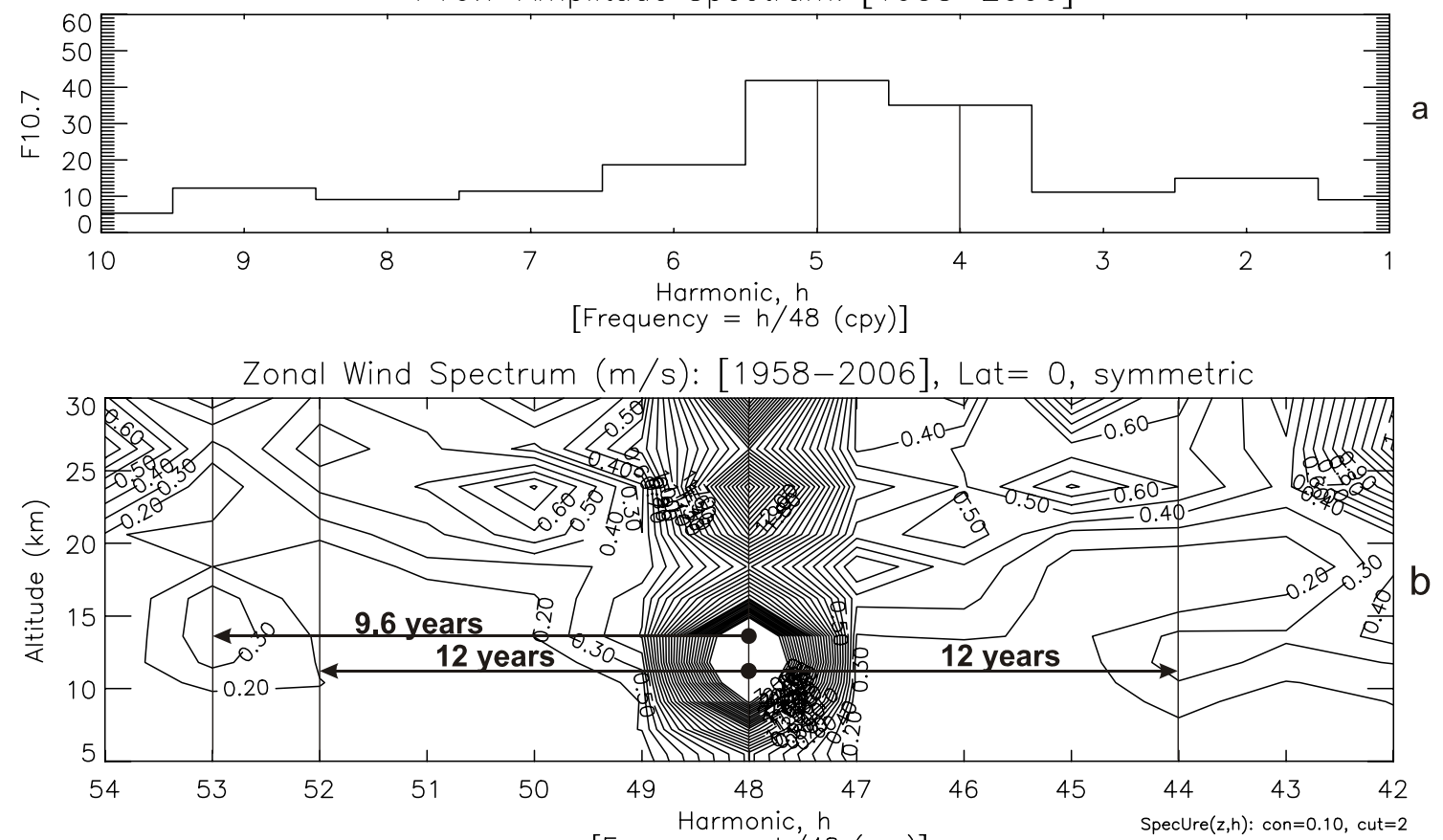

[Frequency $=\mathrm{h} / 48$ (cpy)]

Temperature Spectrum (K): [1958-2006], Lat $=0$, symmetric

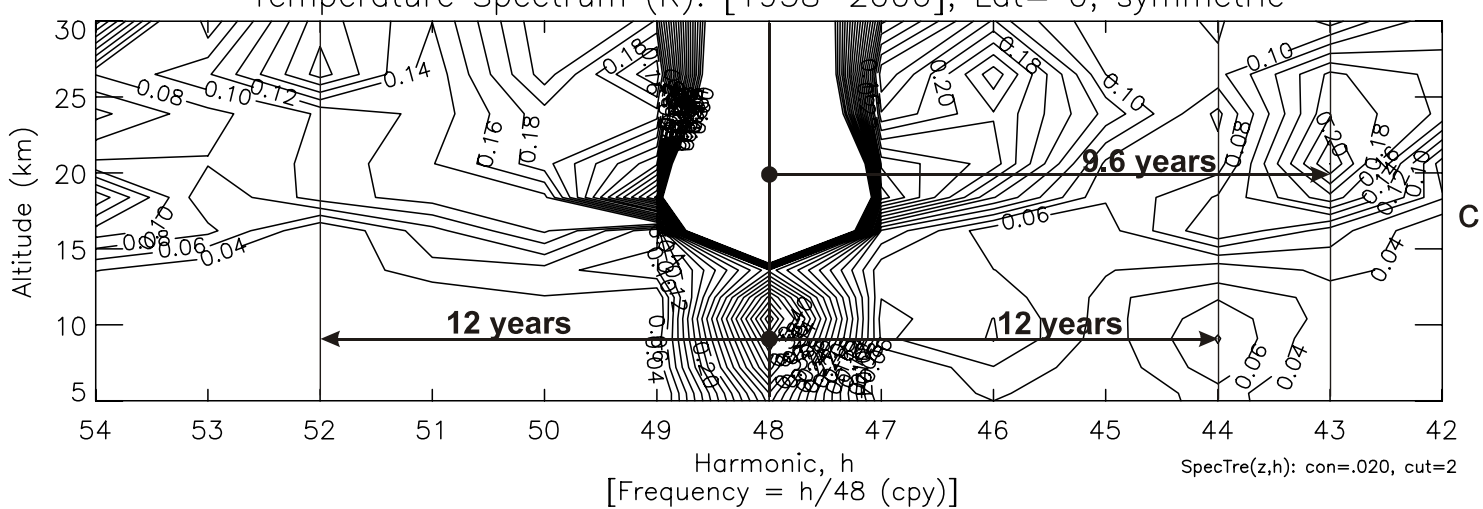

Temperature Spectrum (K): [1958-2006], Lat $=90$, symmetric

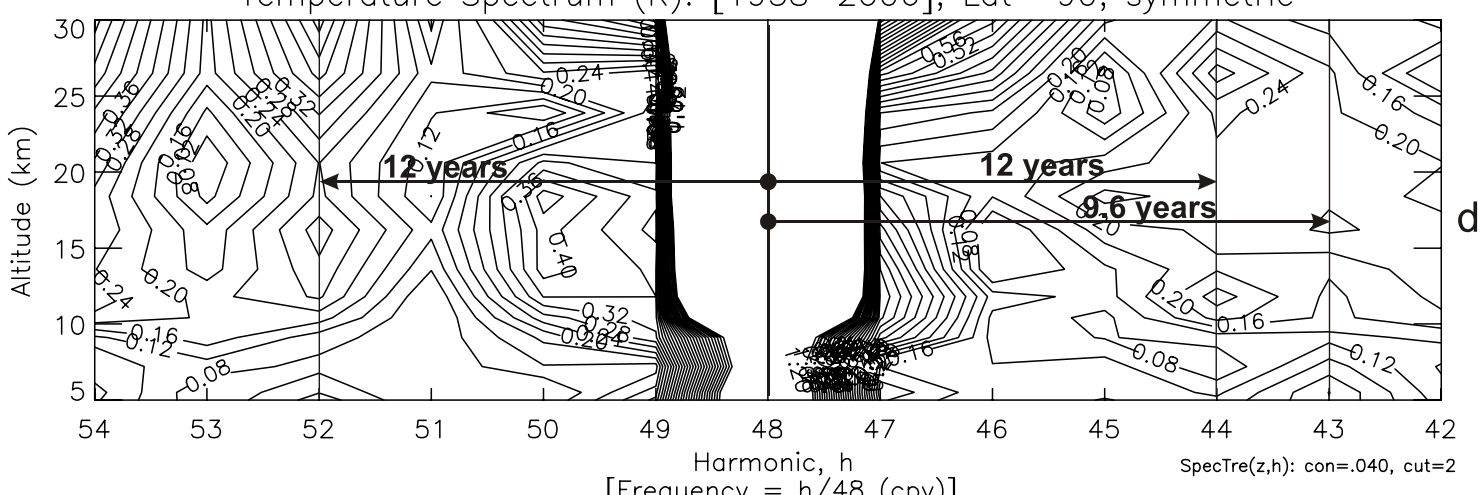

Fig. 6. (a) Spectrum for the F10.7 monthly solar flux from 1958 to 2006 , with the dominant harmonics h=4 and 5 for the periods of 12 and 9.6 months, respectively. (b) Symmetric zonal wind spectrum at the equator for the entire 48-years of NCEP/NCAR data. (c, d) Similar to (b), but showing the symmetric temperature spectra at the equator (c) and pole (d). In the spectra, the modulation periods are indicated with arrows, commensurate with the dominant harmonics for the solar flux in (a). 

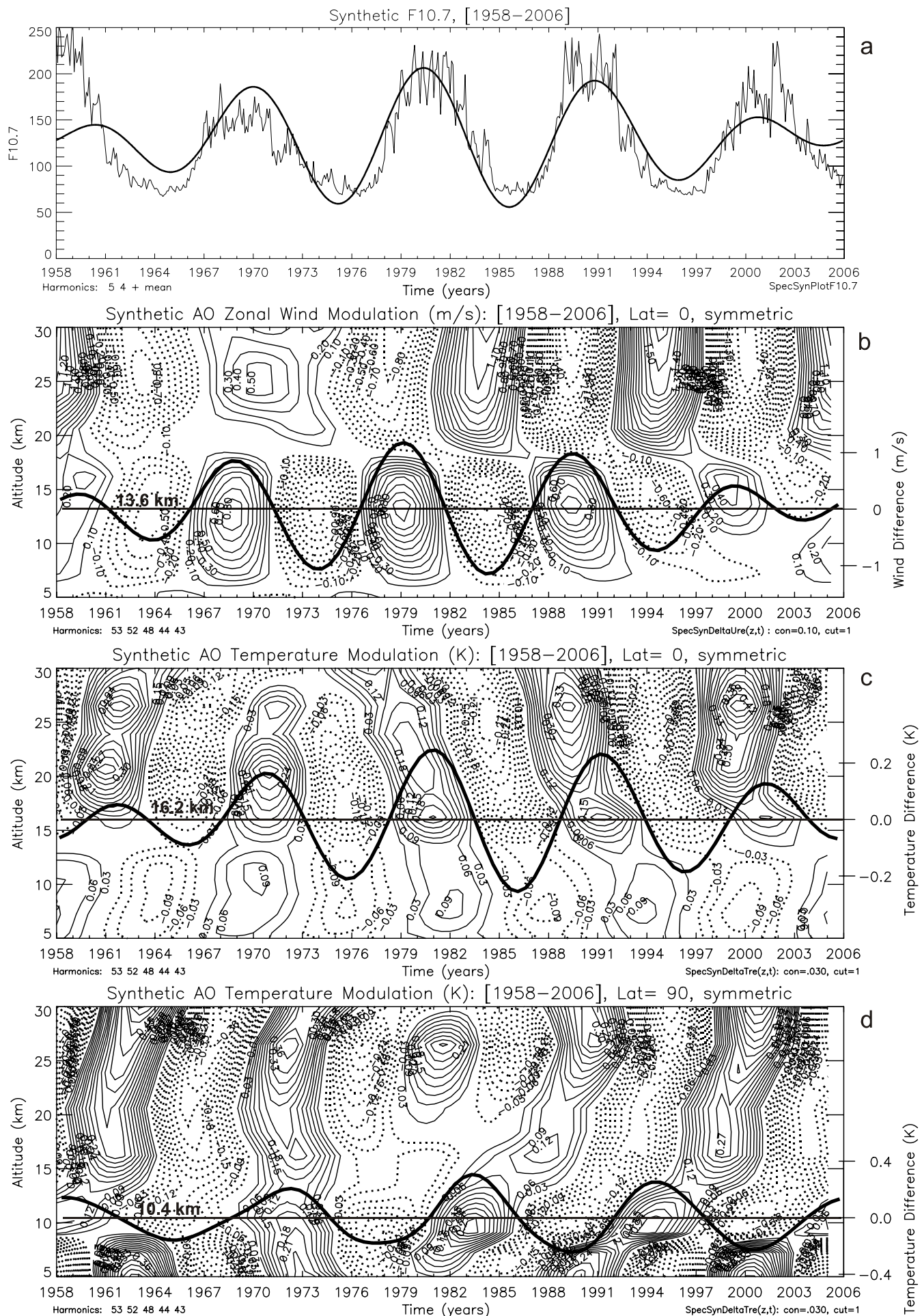

Fig. 7. (a) The monthly $10.7 \mathrm{~cm}$ flux is shown together with synthesized variations employing $\mathrm{h}=4$ and 5 for the periods of 12 and 9.6 years, respectively. (b) Employing $\mathrm{h}=48$ with $\mathrm{h}=(48 \pm 4)$ and $(48 \pm 5)$, synthesized (filtered) modulations of the zonal winds are presented as contour plot at the equator. With heavy solid line, the modulations are shown at $13.6 \mathrm{~km}$ where the phase-shifted pattern is similar to that of the synthesized solar flux variation in (a). (c) Analogous to (b) but synthesized temperature modulations at the equator, and with heavy line showing the pattern at $16.2 \mathrm{~km}$. (d) Synthesized temperature variations at the pole, and with heavy line at $10.4 \mathrm{~km}$. In limited attitude regimes, the filtered equatorial zonal wind (b) and temperature (c, d) modulations of the symmetric AO mimic the solar cycle variability. 
In the Polar Region (Fig. 7d), the synthesized temperature variations above $15 \mathrm{~km}$ have no resemblance to the solar cycle variability. Only at altitudes around $10 \mathrm{~km}$ is there some evidence of a systematic 12-year source, as seen also in the spectrum (Fig. 6d), and the modulations are larger than those at the equator (Fig. 7c).

\section{Conclusion and critique}

In several papers, the solar cycle (SC) signatures in the lower atmosphere have been linked observationally to the QBO zonal winds. Solar cycle variations connected to the QBO are observed at polar latitudes (e.g., Labitzke, 1982; Labitzke and van Loon, 1988; Dunkerton and Baldwin, 1992; Baldwin and Dunkerton, 1998), and the QBO itself is observed varying with the period of 11 years (Salby and Callaghan, 2000, 2006; Hamilton, 2002). The QBO is generated by wave mean flow interactions, which are exceptionally effective at equatorial latitudes where the meridional circulation does not dissipate the flow oscillation (Lindzen and Holton, 1968). Due to the non-linear feedback in the wave momentum source, related to critical level absorption, the SC signatures would be amplified by tapping the momentum from the upward propagating waves.

Analysis of NCEP/NCAR Re-1 data shows that, besides the QBO, a symmetric equatorial 12-month annual oscillation $(\mathrm{AO})$ is observed in the lower stratosphere (Mayr et al., 2007b). Since the SC connection to the atmosphere is likely to occur through the seasonal variations, we asked whether this AO has embedded such a signature. Such an equatorial $\mathrm{AO}$ is generated in a modeling study for the SC modulation of the QBO (Mayr et al., 2005).

Analyzing NCEP/NCAR Re-1 data, we have shown for 44-year moving windows that 11-year signatures appear in the hemispherically symmetric component of the AO, which peak in the zonal winds at equatorial latitudes. At altitudes below $20 \mathrm{~km}$, the synthesized variations are phase locked for 5 consecutive data windows, one year apart, which suggests that the modulations are generated by systematic SC forcing. The similarities in Fig. 7 between the SC variability and the modulations of the $\mathrm{AO}$ zonal winds, and temperatures to a lesser extent, are intriguing. The modulation pattern of the zonal winds near $14 \mathrm{~km}$ (Fig. 7b) mimics, with constant phase difference, the variable maxima and minima of the solar flux index (Fig. 7a). Considering the central importance of wave mean flow interactions around the equator, it is understandable that the SC signatures are more pronounced in the zonal winds. For the temperature variations (Fig. 7c, d), the meridional circulation and planetary waves become additionally involved in redistributing the energy, and this complicates the dynamics, in particular at higher latitudes.

At altitudes above $20 \mathrm{~km}$, the inferred zonal wind and temperature modulations (Figs. 3, 5, 7) are more variable, which indicates that systematic SC forcing is not the prin- cipal agent. This runs contrary to the SC related heat input into the atmosphere, which increases at higher altitudes due to the increasing contribution from the shorter-wavelength radiation. The SC signatures in the NCEP/NCAR Re-1 data thus should get stronger, not weaker as our analysis indicates. The reasons for this could be that at higher altitudes the observations become less reliable, and/or the data coverage deteriorates. With the relatively weak SC forcing, destructive interference could be another reason. The observed trend of the height variations for the inferred 11-year variations of the symmetric $\mathrm{AO}$, however, does pose a problem for the SC interpretation proposed in this paper.

Acknowledgements. The authors are indebted to an anonymous referee whose comments contributed significantly to improve the paper.

Topical Editor C. Jacobi thanks E. Kazimirovsky and another anonymous referee for their help in evaluating this paper.

\section{References}

Baldwin, M. P. and Dunkerton, T. J.: Biennial, quasi-biennial, and decadal oscillations of potential vorticity in the northern stratosphere, J. Geophys. Res., 103, 3919-3928, 1998.

Cordero, E. C. and Nathan, T. R.: A new pathway for communicating the 11-year solar cycle to the QBO, Geophys. Res. Lett., 32, L18805, doi:10.1029/2005GL023696, 2005.

Dunkerton, T. J. and Baldwin, M. P.: Modes of interannual variability in the stratosphere, Geophys. Res. Lett., 19, 49-51, 1992.

Hamilton, K.: On the Quasi-decadal modulation of the stratospheric QBO period, J. Climate, 15, 2562-2565, 2002.

Haynes, P. H.: The latitudinal structure of the quasi-biennial oscillation, Q. J. Roy. Meteorol. Soc., 124, 2645-2670, 1998.

Hines, C. O.: Doppler-spread parameterization of gravity-wave momentum deposition in the middle atmosphere, 1 , Basic formulation, J. Atmos. Solar Terr. Phys., 59, 371-386, 1997 a.

Hines, C. O.: Doppler-spread parameterization of gravity-wave momentum deposition in the middle atmosphere, 2, Broad and quasi monochromatic spectra, and implementation, J. Atmos. Solar Terr. Phys., 59, 387-400, 1997b.

Hocke, K.: Oscillation of global mean TEC, J. Geophys., Res., 113, A04302, doi:10.1029/2007JA0123798, 2008.

Huesmann, A. S. and Hitchman, M. H.: The 1978 shift in the NCEP reanalysis stratospheric quasi-biennial oscillation, Geophys. Res. Lett., 30, 1048, doi:10.1029/2002GL016323, 2003.

Huesmann, A. S. and Hitchman, M. H.: The stratospheric quasibiennial oscillation in the NCEP reanalysis: Climatological structure, J. Geophys. Res., 106, 11859-11874, 2001.

Kalnay, E.: Atmospheric modeling, data assimilation and predictability, Cambridge University Press, Cambridge, UK, 2003.

Kalnay, E., Kanamitsu, M., Kistler, R., et al.: The NCEP/NCAR 40-year reanalysis project, B. Am. Meteorol. Soc., 77, 437-471, 1996.

Labitzke, K.: On the inter-annual variability of the middle stratosphere during northern winters, J. Meteorol. Soc. Jpn., 60, 124139, 1982.

Labitzke, K.: Sunspots, the QBO and stratospheric temperature in the north polar region, Geophys. Res. Lett., 14, 535-537, 1987. 
Labitzke, K. and Van Loon, H.: Association between the 11-year solar cycle, the QBO and the atmosphere. Part I: the troposphere and stratosphere in the northern hemisphere in winter, J. Atmos. Terr. Phys., 50, 197-206, 1988.

Labitzke, K. and Van Loon, H.: On the association between the QBO and the extratropical stratosphere, J. Atmos. Terr. Phys., 54, 1453-1463, 1992.

Lindzen, R. S. and Holton, J. R.: A theory of the quasi-biennial oscillation, J. Atmos. Sci., 25, 1095-1107, 1968.

Matthes, K., Langematz, U., Gray, L., Kodera, K., and Labitzke, K.: Improved 11-year solar signal in the Freie Universitaet Berlin, Limate Middle Atmosphere Model (FUB-CMAM), J. Geophys., Res., 109, D06101, doi:10.1029/2003JD004012, 2004.

Mayr, H. G., Mengel, J. G., and Wolff, C. L.: Wave-driven equatorial annual oscillation induced and modulated by the solar cycle, Geophys. Res. Lett., 32, L20811, doi:101029/2005GL023090, 2005.

Mayr, H. G., Mengel, J. G., Wolff, C. L., and Porter, H. S.: QBO as potential amplifier of solar cycle influence, Geophys. Res. Lett., 33, L05812, doi:10.1029/2005GL025650, 2006.

Mayr, H. G., Mengel, J. G., Wolff, C. L., Huang, F. T., and Porter, H. S.: The QBO as potential amplifier and conduit to lower altitudes of solar cycle influence, Ann. Geophys., 25, 1071-1092, 2007a, http://www.ann-geophys.net/25/1071/2007/.
Mayr, H. G., Mengel, J. G., Huang, F. T., and Nash, E. R.: Equatorial annual oscillation with QBO-driven 5-year modulation in NCEP data, Ann. Geophys., 25, 37-45, 2007b, http://www.ann-geophys.net/25/37/2007/.

Mayr, H. G., Mengel, J. G., Huang, F. T., Talaat, E. R., Nash, E. R., and Reddy, C. A.: Stratospheric semi-decadal oscillations in NCEP data, Ann. Geophys., 26, 2143-2157, 2008, http://www.ann-geophys.net/26/2143/2008/.

Mengel, J. G., Mayr, H. G., Chan, K. L., Hines, C. O., Reddy, C. A., Arnold, N. F., and Porter, H. S.: Equatorial oscillations in the middle atmosphere generated by small scale gravity waves, Geophys. Res. Lett., 22 , 3027-3039, 1995.

Palmer, M. and Gray, L.: Modeling the atmospheric response to solar irradiance changes using a GCM with a realistic QBO, Geophys. Res. Lett., 32, L24701, doi:10.1029/2005GL023809, 2005.

Salby, M. and P. Callaghan, P.: Connection between the solar cycle and the QBO: The missing link, J. Climate, 13, 2652-2662, 2000.

Salby, M. and Callaghan, P.: Relationship of the quasi-biennial oscillation in the stratospheric signature of the solar cycle, J. Geophys. Res., 111, D06110, doi:10.1029/2005JD006012, 2006. 